\title{
An Adaptive Tracker for Assisted Living
}

\author{
Domenico Bloisi, Luca Iocchi, Luca Marchetti \\ Dipartimento di Informatica e Sistemistica \\ Sapienza University of Rome \\ \{bloisi, iocchi, lmarchetti\}@dis.uniromal.it
}

\author{
Dorothy N. Monekosso \\ Centre for Adaptive Wireless Systems \\ Cork Institute of Technology \\ dorothy.monekossodcit.ie
}

\author{
Paolo Remagnino \\ Kingston University London \\ Surrey, U.K \\ p.remagninoekingston.ac.uk
}

\begin{abstract}
We propose an adaptive tracking system for assisted living that integrates user information about emergency events. Information fusion between user data and visual data is performed in order to estimate and assess the situation at hand. The system is able to dynamically switch between different segmentation and tracking algorithms improving its performance, as shown by the proposed examples.
\end{abstract}

\section{Introduction}

Visual tracking in dynamic and complex scenes is one of the most challenging research topics in Computer Vision.

Ambient Assisted Living (AAL) environments will increasingly allow elderly people to remain active and productive for longer, to continue and engage in the society with more accessible on line services, and to enjoy a healthier and higher quality of life for longer. Examples of applications about safety and security are accident monitoring, visitor validation and activity detection.

In this paper we propose an adaptive tracking system for assisted living able to integrate information from a user about the scene context. Due to sensor noise, motion in the scene, occlusions and real-time constraints the objective of automatically detecting and tracking objects of interest is a hard task. Using a human operator as a dynamic control agent, that can provide updated contextual information about the observed environment, can be exploited to improve the system performance. Information fusion between user data and visual data is performed in order to estimate and assess the situation at hand.
The paper is organized as follows. After discussing related work in Section 2, the general architecture of the system is described in Section 3. In Section 4 and 5 we present the segmentation and tracking modules respectively. The data fusion layer is detailed in Section 6. Section 7 shows results obtained by our approach, while Section 8 concludes the paper.

\section{Related Work}

Assisted living systems can be divided in non-visual and visual systems [7]. The former class is made of wireless sensor systems using RFID, GPS, infrared tags, accelerometers and pressure pads without visual information from cameras. Examples of such systems are CodeBlue [10] (a wireless infrastructure intended for the deployment in emergency medical care), the Assisted Cognition Project [9] (that aims to develop novel computer systems that will enhance the quality of life of people suffering from Alzheimers Disease and similar cognitive disorders) and ALARM-NET [13] (a wireless sensor network for assistedliving and residential monitoring).

Currently used worn sensors may produce false alarms, elderly people can forget wearing them $[12,7]$ and medical staff often prohibits any wearable sensors as well as devices that could not be concealed from patients or that require patient approval [8].

Computer vision based systems propose non-invasive alternatives for monitoring a scene, but suffer from inherent problems such as occlusions, illumination changes, shadows and tracking failures in crowded environments.

Examples of visual systems are CareMedia [8] (performing long-term observation of the elderly in nursing homes), SmartClassySurv [7] and [12] (both of them are systems for 
falling person detection).

We propose a novel approach based on a data fusion process between hard electronically-based observational and soft human generated data in order to cope with the complexity of the scenarios in which automatic surveillance systems will have to operate.

\section{Project Overview}

The system monitors the training of student nurses in the School of Nursing of the Faculty of Health and Social Care Sciences at Kingston University, London. The aim is to detect and track people in order to analyze their behavior and feed back useful information to the instructors.

The general architecture of the approach is illustrated in Fig. 1. It is composed of four modules: segmentation, tracking, data fusion and event handling.

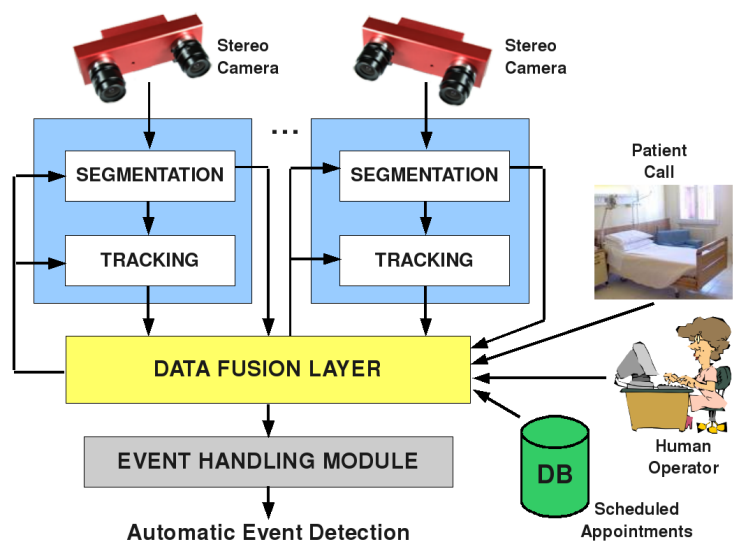

Figure 1. General architecture of the system.

Data are collected using two commercial stereo cameras (Videre Design STH-MDCS2) each camera connected to a computing unit (an Intel Core 2 Duo 2,0 GHz CPU Mac mini) through a Firewire connection.

Each computing unit receives data from the stereo camera performing segmentation and tracking in a local coordinate system (see top of Fig. 1).

The data fusion layer is wirelessly connected to the computing unit in order to input information about the segmentation and tracking algorithms to use according to: 1) the data received from a human operator, 2) a database storing scheduled appointments and 3) patient calls. The data fusion layer also merges information from the tracking modules, transforming from local coordinates to a global coordinate system.

The event handling module displays images from the deployed cameras, builds a ground plane view (see Fig.7) and records events of interest. In the following, we will discuss two particular events, in order to highlight the use of context information in improving system performance:
- a nurse assisting a patient for a scheduled appointment, e.g. an injection (see Fig. 3);

- a team of medical doctors visiting a patient (see Fig. 4 and Fig. 5).

\section{Segmentation}

The segmentation module architecture is detailed in Fig. 2. The background estimate step creates a background model of the observed scene exploiting both color (left image) and depth information (disparity image) from the stereo camera. The foreground extraction process uses background the subtraction technique to create the foreground image representing moving objects. All the details about the two above mentioned steps can be found in [5].

Contextual information is used by the segmentation module to switch between two different algorithms (see Filter Switching module in the center of Fig. 2).

Information about the number of people that enters the scene is collected in an on-line mode (human operator and patient calls) or exploiting a scheduled appointments database. If the expected number of people in the scene provided by Data Fusion module is less than 3, the Algorithm 1 called "Rek-means" is used, otherwise the Algorithm 2, called "Height Image Algorithm" becomes active.

Rek-means [3] exploits the pyramidal Lukas-Kanade Optical Flow (OF) computation [6] (that yields a sparse map) and the K-means based data clustering to segment moving objects. OF gives information about moving directions and can be used for solving over-segmentation (e.g. shadows on walls and furniture produced by people as well as false positives produced by moving beds and room screens can be filtered since they are associated with a negligible number of OF points) and under-segmentation (two people moving in opposite directions generate opposite OF vector fields). Rek-means takes as input the OF sparse map and clusters its points according to an iterative algorithm (see Fig. 3).

The above presented algorithm cannot be used when the scene becomes crowded due to noise in the OF map (compare OF images in Figures 3, 4 and 5).

The Height Image Algorithm (HI) [5] is designed for detecting up to 15 people in the scene simultaneously. Instead of OF, HI exploits the Sobel's operator for edge detection and the frame edge temporal differencing for individuating "active" regions of the scene. Such regions are analyzed in order to segment the exact number of people in the scene.

\section{Tracking}

The result of the segmentation process is a set of 3D points representing the heads of detected people. Tracking is performed based on ground-plane projection of such 


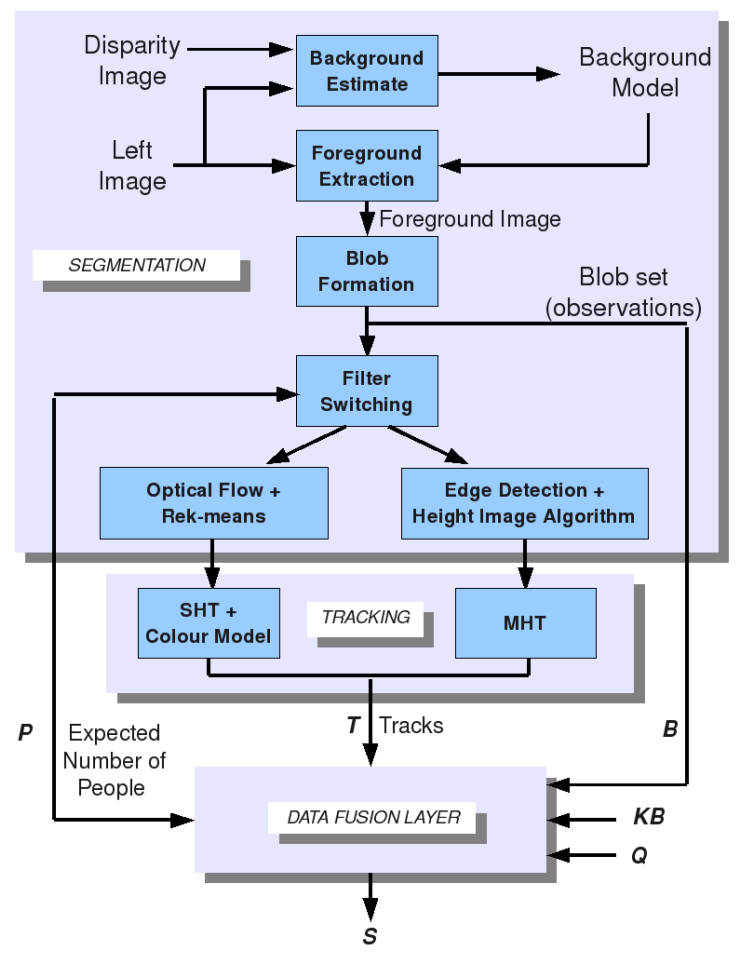

Figure 2. Segmentation and Tracking module details. The system is able to switch between different segmentation and tracking algorithms depending on context information and observations.

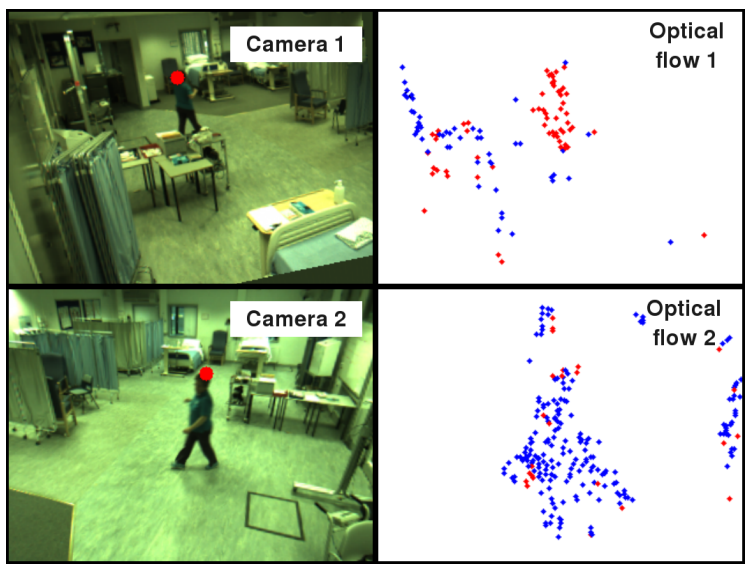

Figure 3. Segmentation using optical flow example - Nurse assisting patient. In optical flow images, red dots represent pixels moving right while blue dots pixels moving left (with respect to the camera field of view).

points (see Fig. 7).

We use a Kalman Filter (KF) to solve the problem of estimating the movements of each object. While KF is used to track a single object (Single Hypothesis Tracking, SHT), when developing a multi-object tracking (Multiple Hypoth-

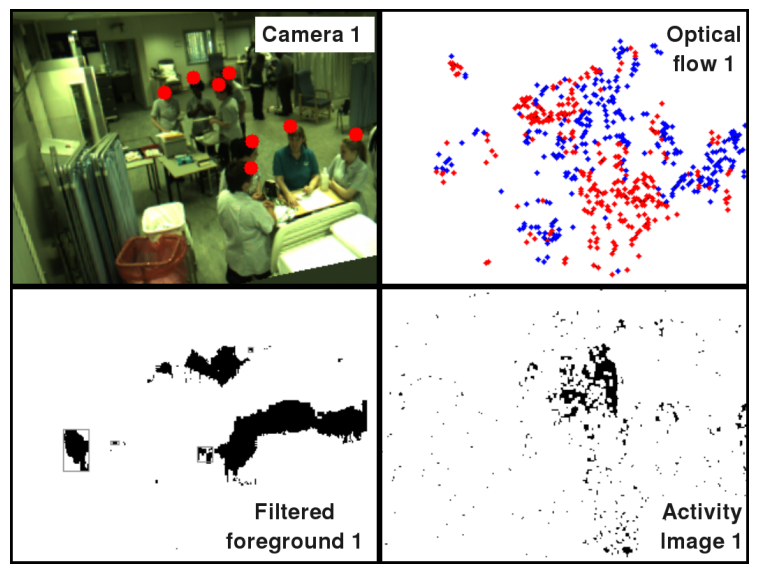

Figure 4. Segmentation using Height Image Algorithm example Group of doctors (camera 1). Rectangles in filtered foreground image represent discarded moving objects due to negligible activity. In particular the room screen in the bottom left corner is detected as a moving object by background subtraction but is filtered out by the activity image analysis.

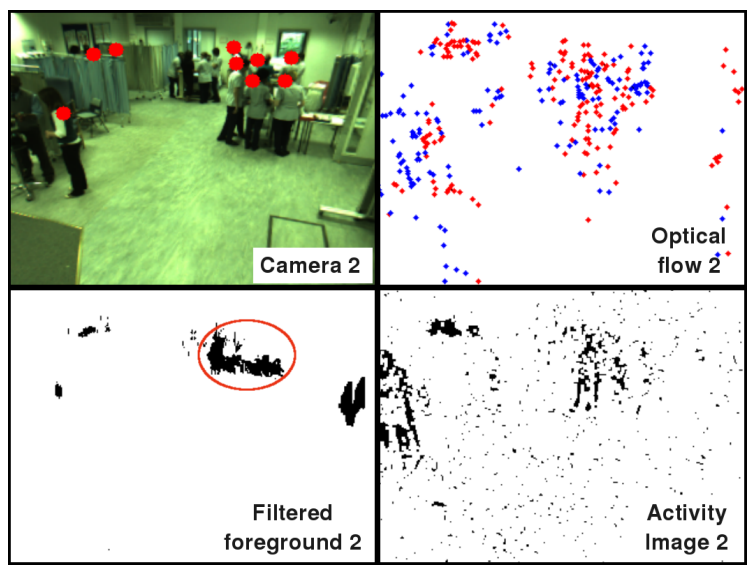

Figure 5. Segmentation using Height Image Algorithm example Group of doctors (camera 2). From the single blob in the red circle it is possible to detect six people.

esis Tracking, MHT) method, one usually has to deal with data association and track management. In our application, especially when we have a very crowded scene, data association is very challenging, since it is not straightforward to assign an observation to a certain track.

As we can retrieve information about the number of people that enters the room, we choose to implement an adaptive tracking algorithm (see Tracking module details in Fig. 2).

When one or two people are in the scene, we apply a SHT algorithm creating a color model for the tracked person. This model and movement directions extracted from OF map are used as additional sources of information for the data association step. Details about the employed SHT 
approach for creating the color model can be found in [1] and some examples in [5].

Building a color model for a tracked object in a crowded situation is an hard task due to occlusions causing noise in the updating of the color model. Thus, when there are more than two people in the scene, we adopt a MHT based on a bank of Kalman Filters. Data association is used to determine the relationships between observations and tracks, but multiple hypotheses are maintained when observations may be associated to more than one track. Indeed an approach based on a single hypothesis is not adequate, since it cannot recover from data association errors. Details about used MHT approach can be found in [4].

\section{Data Fusion}

The data Fusion layer collects all information from all camera devices, the appointments database, the human operator, the hospital schedule and returns a probability assessment on possible situations.

The fusion process builds a representation of the environment, describing the people occupying rooms and corridors. The basic idea is to assign an information profile to each track, describing the role and the purpose related to the situation.

A sketch of the algorithm is given in Algorithm 1.

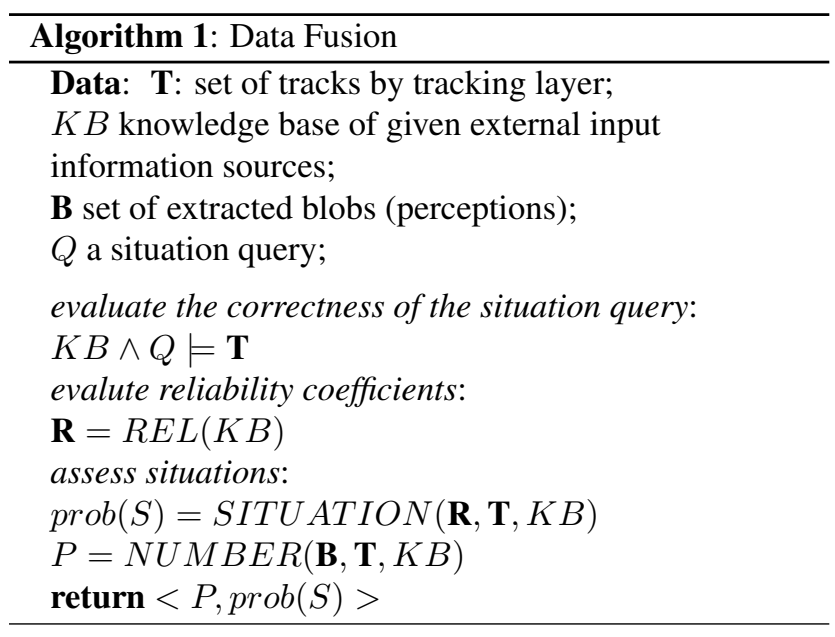

The first step consists of a predicted estimation of association between people and rooms. Given the information about appointments, the working hours of the employees, the visiting hours and the scheduled medications, it is possible to determine the category of people present in a specific area of the environment.

The second step performs the situation assessment. It uses the contextual information provided by the previously defined sources, and returns a probability evaluation of possible situations.
To exploit the a priori information from different sources, a reliability value is associated to each information source [11]. For example, a human operator will introduce in the system the most reliable information: he can identify correctly whether a person is a nurse, a doctor or a patient. The visiting hours, instead, will give less reliable information: the presence of patient's relative is possible even outside the visiting hours. These reliability coefficients are used to weight the importance of information during the situation assessment.

To evaluate the Reliability Coefficients $\mathbf{R}$, let us assume a feature vector $\mathbf{F}$. This describes the characteristics of each source as follows:

- failure rate: percentage of erroneous classification;

- consistency: agreement with different information sources;

- situation confidence: ability to detect given situation.

Using a Mahalanobis distance operator, each information source's vector is compared with a situation $S$ from the set of situations $\mathbf{S}$. The results is a probability value that the given tracks support the situation $S$.

\section{Implementation and Example}

The presented system is an integration between existing segmentation and tracking software modules designed by the authors. Such an integration is possible thanks to the newly developed Data Fusion layer that aims to exploit soft data coming from user interaction and hard data from stereo cameras.

In Fig. 6 an example of data fusion is presented. In frame 1 the system receives contextual information from the user about an event of emergency involving 4 nurses. The system performs SHT with color model (see rectangles around detected people) until frame 3 when the detected number of people in the scene becomes equal to 3 . Since the system knows the expected number of people will be 4, it switches to MHT without color model (no rectangles around people).

Using a SHT approach works well in the case of widely spaced targets (e.g. one or two people in the scene), accurate measurements and few false alarms, but can fail with a large number of observations because misassociations cannot be recovered and lead to track loss and error in prediction [2].

Using a MHT approach is useful in crowded environments, but can generate too many tracks with respect to the real number of people in the scene when there are few observations because of the creation of new tracks for unassociated observations that can be produced by noise in the segmentation step.

Our adaptive approach permits to exploit advantages of both SHT and MHT. 


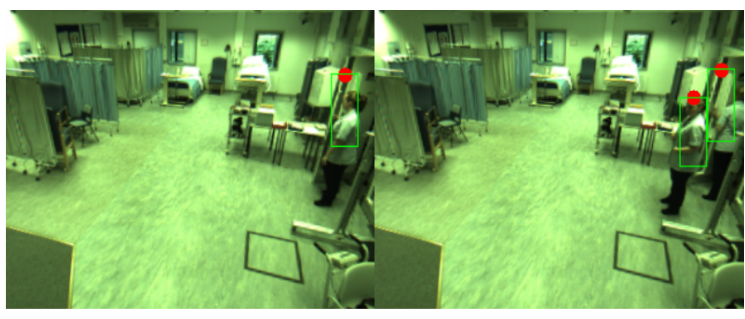

1

2

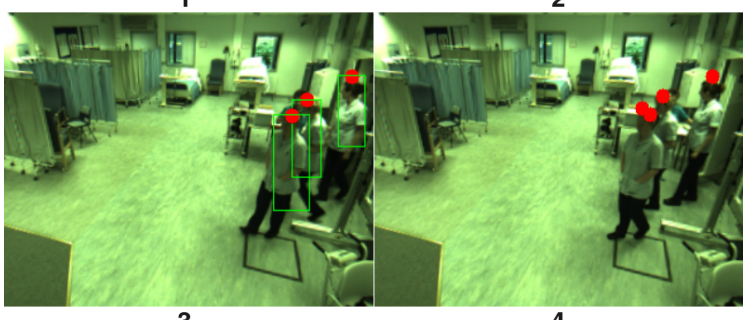

3 4

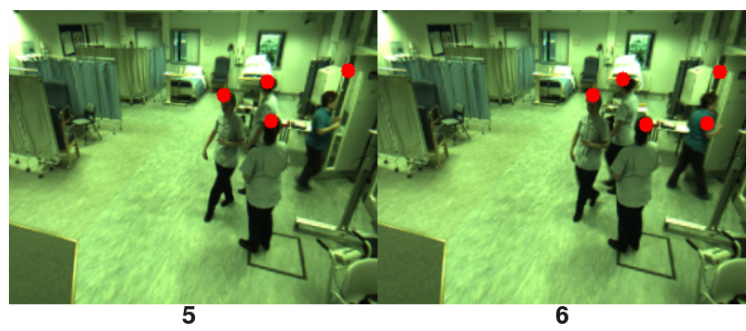

Figure 6. Example of an on-line switch between different tracking algorithms.

Data fusion procedure for situation assessment completes the system framework (see Fig. 2). In Fig. 8 an execution of algorithm 1 in a complex scenario is shown. At time $\mathbf{A}$ a nurse enters into the room (Fig. 3) for a puncture, while at time $\mathbf{B}$ and $\mathbf{C}$ a group of doctors come for a visit (Fig. 4).

The graphs show the probability associated with the corresponding situation. We classified the situation according to the output of the tracking algorithm and using global knowledge from user interaction and from scheduled appointments database. The system is able to correctly classify both the situations: the nurse (A) and the group of doctors (B and $\mathbf{C})$.

\section{Conclusions}

A video surveillance system for monitoring a nursing environment has been presented. An adaptive tracking algorithm permits to detect and track nurses and patients in their activity. The main novelty consists in the use of soft data coming from user interaction that are managed by a data fusion module in order to drive and adapt the tracking module to the best tracking policy. An example of switching between different tracking strategies is shown highlighting benefits of an adaptive approach. As future works we in-
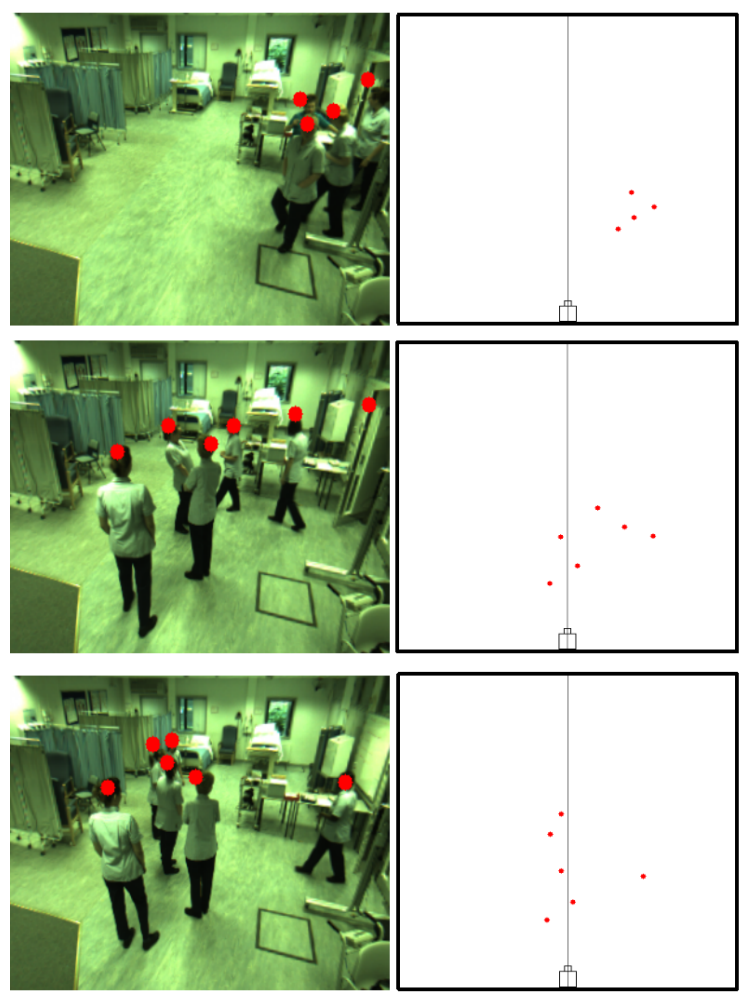

Figure 7. Example of a ground plane view transformation.

tend to evaluate the approach on a set of predefined sistuations and to add a crowd flow analysis module in order to improve tracking.

\section{References}

[1] S. Bahadori, L. Iocchi, G. R. Leone, D. Nardi, and L. Scozzafava. Real-time people localization and tracking through fixed stereo vision. Applied Intelligence, 26(2):83-97, 2007.

[2] S. S. Blackman. Multiple hypothesis tracking for multiple target tracking. Aerospace and Electronic Systems Magazine, 19(1):5-18, 2004.

[3] D. D. Bloisi and L. Iocchi. Rek-Means: A k-Means Based Clustering Algorithm. In Computer Vision Systems, 6th International Conference (ICVS 2008), LNCS 5008, pages 109-118, 2008.

[4] D. D. Bloisi, L. Iocchi, G. R. Leone, R. Pigliacampo, L. Tombolini, and L. Novelli. A distributed vision system for boat traffic monitoring in the venice grand canal. In Proc. of 2nd Intern. Conf. on Computer Vision Theory and Applications (VISAPP-2007), pages 549-556, 2007.

[5] D. D. Bloisi, L. Iocchi, D. N. Monekosso, and P. Remagnino. A novel segmentation method for crowded scenes. In Proc. of 4th Intern. Conf. on Computer Vision Theory and Applications (VISAPP-2009), pages 484-489, 2009.

[6] J. Y. Bouguet. Pyramidal implementation of the lucas kanade feature tracker: description of the algorithm, 2002. 


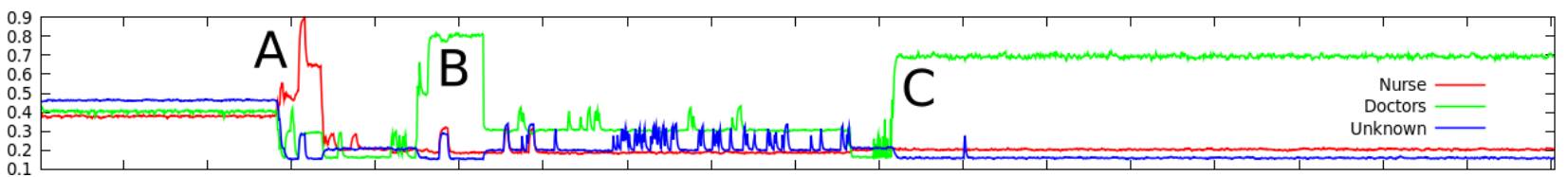

Figure 8. An example of complete working framework. At time $\mathbf{A}$ a nurse enter into the room, while at time $\mathbf{B}$ and $\mathbf{C}$ a group of doctors enter for a visit. The graphs show the probability associated to each situation. The system is able to detect correctly the situation. The probability of unknown situation is given by blue line.

[7] S. Fleck, R. Loy, C. Vollrath, F. Walter, and W. Strasser. Smartclassysurv - a smart camera network for distributed tracking and activity recognition and its application to assisted living. In First ACM/IEEE International Conference on Distributed Smart Cameras, pages 211-218, 2007.

[8] A. Hauptmann, J. Gao, R. Yan, Y. Qi, J. Yang, and H. Wactlar. Automated analysis of nursing home observations. In IEEE Pervasive Computing, volume 3, pages 15-21, 2004.

[9] H. Kautz, D. Fox, O. Etzioni, G. Borriello, and L. Arnstein. An overview of the assisted cognition project. In AAAI-2002 Workshop on Automation as Caregiver: The Role of Intelligent Technology in Elder, 2002.

[10] D. Malan, T. Fulford-Jones, M. Welsh, and S. Moulton. Codeblue: An ad hoc sensor network infrastructure for emergency medical care. In International Workshop on Wearable and Implantable Body Sensor Networks, 2004.

[11] L. Marchetti, D. Nobili, and L. Iocchi. Improving tracking by integrating reliability of multiple sources. In Information Fusion, 2008 11th International Conference on, pages 1-8, 30 2008-July 32008.

[12] B. U. Toreyin, Y. Dedeoglu, and A. E. Cetin. Hmm based falling person detection using both audio and video. In Computer Vision in Human-Computer Interaction, pages 211220, 2005.

[13] A. Wood, G. Virone, T. Doan, Q. Cao, L. Selavo, Y. Wu, L. Fang, Z. He, S. Lin, and J. Stankovic. Alarm-net: Wireless sensor networks for assisted-living and residential monitoring. Technical report, Department of Computer Science, University of virginia, 2006. 